\title{
Inflammation-related genes S100s, RNASE3, and $C Y B B$ and risk of leukemic transformation in patients with myelodysplastic syndrome with myelofibrosis
}

Minghua Hong ${ }^{1 \dagger}$, Junqing Wu ${ }^{1 \dagger}$, Lifeng $\mathrm{Ma}^{2}$, Xiaoping $\mathrm{Han}^{2}$, Ting Lu' ${ }^{1}$, Zhaoming Wang ${ }^{3}$, Jing Zhao ${ }^{3}$, Lizhen Liu', Huarui Fu', Weijia Huang ${ }^{1}$, Weiyan Zheng ${ }^{1}$, Jingsong He${ }^{1}$, Guoqing Wei ${ }^{1}$, Huanping Wang ${ }^{1}$, Zhimei Chen ${ }^{1}$, He Huang ${ }^{1}$, Zhen Cai ${ }^{1}$ Guoji Guo ${ }^{2 \dagger}$ and Jie Sun ${ }^{1 *+}$

\begin{abstract}
Myelodysplastic syndrome with myelofibrosis (MDS-MF) has been associated with an inferior prognosis compared with MDS without MF. However, MDS-MF is not listed independently as a subtype of MDS, and its clinical and genetic characteristics remain poorly understood. We retrospectively compared 53 patients with MDS-MF (44 MF grade $1 / \mathrm{MF}_{1} ; 9 \mathrm{MF}$ grade $2-3 / \mathrm{MF}_{2-3}$ ) and 31 with de novo MDS without MF (MDS). The leukemic transformation risks of both $M D S-M F_{2-3}$ and MDS-MF 1 were increased compared with the MDS group. To identify the potential mechanisms responsible for the leukemic transformation of MDS-MF, we performed single-cell sequencing for one MDS-MF ${ }_{2-3}$ patient before and after leukemic transformation to explore the variations in gene expression levels. In addition to upgraded expression levels of acute myeloid leukemia-related genes during leukemic transformation, expression levels of some inflammation-related genes (such as S100s, RNASE3, and CYBB) were also increased, and inflammation-related pathways were up-regulated. These results suggest that inflammation-related genes and pathways may play an important role in the leukemic transformation of MDS-MF.
\end{abstract}

Keywords: Myelodysplastic syndrome, Myelofibrosis, Leukemic transformation, Single-cell sequence, Inflammation

\footnotetext{
*Correspondence: jsun1492@zju.edu.cn

${ }^{\dagger}$ Minghua Hong and Junqing Wu, Guoji Guo and Jie Sun contributed equally to this work.

'Bone Marrow Transplantation Center, the First Affiliated Hospital, Zhejiang University School of Medicine; Institute of Hematology, Zhejiang University; Zhejiang Province Engineering Laboratory for Stem Cell and Immunity

Therapy; Liangzhu Laboratory, Zhejiang University Medical Center, 1369 West Wenyi Road, 310003 Hangzhou, China

Full list of author information is available at the end of the article
}

\section{$\triangle B M C$}

(C) The Author(s). 2021 Open Access This article is licensed under a Creative Commons Attribution 4.0 International License, which permits use, sharing, adaptation, distribution and reproduction in any medium or format, as long as you give appropriate credit to the original author(s) and the source, provide a link to the Creative Commons licence, and indicate if changes were made. The images or other third party material in this article are included in the article's Creative Commons licence, unless indicated otherwise in a credit line to the material. If material is not included in the article's Creative Commons licence and your intended use is not permitted by statutory regulation or exceeds the permitted use, you will need to obtain permission directly from the copyright holder. To view a copy of this licence, visit http://creativecommons.org/licenses/by/4.0/. The Creative Commons Public Domain Dedication waiver (http://creativecommons.org/publicdomain/zero/1.0/) applies to the data made available in this article, unless otherwise stated in a credit line to the data. 
To the Editor

Myelodysplastic syndrome (MDS) with myelofibrosis (MDS-MF) accounts for up to $50 \%$ of cases of MDS [1], and differs from MDS without MF in terms of clinical performance, treatment tolerance, and survival [2]. MDS with severe MF (MF grade $=2-3$ ) is considered as an independent risk factor for de novo MDS [3]; however, MDS-MF is not independently listed as a subtype of MDS according to the 2016 World Health Organization classification, indicating the need for further investigation.

We retrospectively enrolled 53 patients with MDS-MF (44 MF grade $1 / \mathrm{MF}_{1} ; 9 \mathrm{MF}_{2-3}$ ) and 31 patients with de novo MDS without MF (MDS). There were no significant differences among the MDS, MDS-MF 1 , and MDS$\mathrm{MF}_{2-3}$ groups in terms of age, sex, MDS subtypes, IPSS risk levels, and treatment strategies, except for a higher rate of poor karyotypes in the $\mathrm{MDS}-\mathrm{MF}_{2-3}$ compared with the MDS and MDS-MF ${ }_{1}$ groups (with no difference between the MDS and MDS-MF ${ }_{1}$ groups) (Supplementary Table 1 ). Not only MDS-MF $\mathrm{MF}_{2}$, but also MDS-MF at IPSS low/int-1 risk, had a shorter leukemic transformation time compared to the MDS group (Fig. 1), suggesting that patients with low/int-1 MDS with even mild MF required chemotherapy to avoid disease progression. Detailed patients' clinical data are shown in Supplementary file 1.

Cytogenetic abnormalities or genomic mutations are related to the leukemic transformation of MDS-MF but cannot account for the role of MF in this process. The leukemia clonal revolution is influenced by many factors in the bone marrow (BM) microenvironment, including inflammation and abnormal immunity. Chronic inflammation is believed to promote malignant hematopoiesis in myeloproliferative neoplasms through pro-inflammatory / fibrogenic /angiogenic cytokines [4-6]. Reactive oxygen species also play a major role in tumor progression of myeloproliferative neoplasms [7]. However, the processes responsible for the clonal myeloproliferation of MDS-MF remain unclear. It is therefore necessary to determine the variations in gene expression levels responsible for the leukemic transformation of MDS-MF. We conducted single-cell sequencing of BM mononuclear cells (BMMCs) from a patient with $\mathrm{MDS}-\mathrm{MF}_{2-3}(\mathrm{CN})$ in the MDS phase (CN1) and leukemic phase (CN2). A healthy donor (NC) and a patient with de novo acute myeloid leukemia (AML) with the same FAB subtype (M2) were used as controls. Patients' clinical information and detailed methods are provided in Supplementary File 1. Cell clusters were identified based on 13,280 healthy cells and BMMCs from 40 patients with newly diagnosed AML in our previous study [8] and were checked with the Human Cell Landscape (http://bis.zju.edu.cn/HCL/index.html) established by our institute [9]. Marker genes for the cell clusters are listed in Supplementary Table 2.

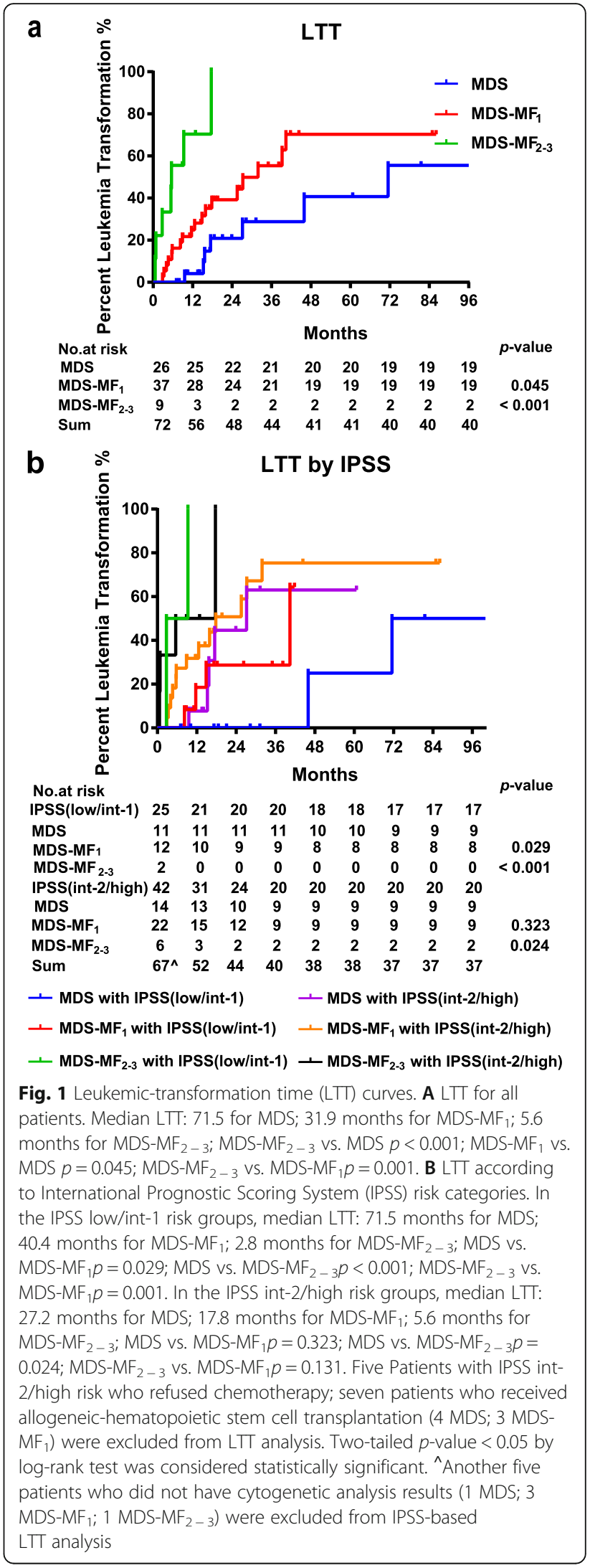



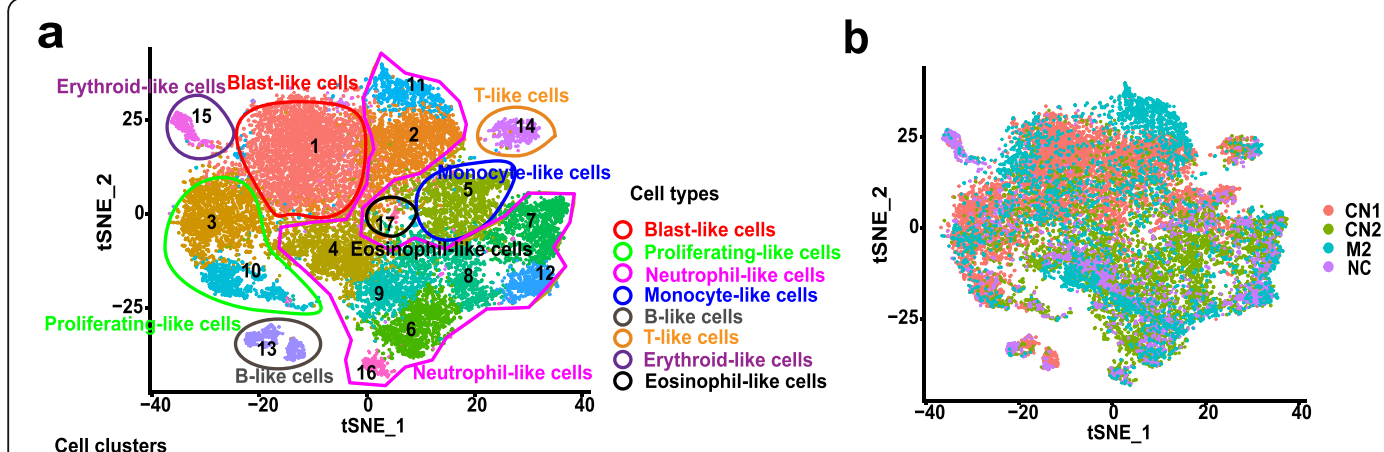

1 Blast-like cells

- 2 Neutrophil-like_S100A10 high cells 10 Proliferating-like cell_UBE2C high cells - 4 Proutiferating-like cell_STMN1 high cells 11 Neutrophil-like_TPSB2 high cells 5 Monocyte-like cells

6 Neutrophil-like_DEFA3 high cells

7 Neutrophil-like_S100A12 high cells
Neutrophil-like_S100A8 high cells

12 Neutrophil-lik

13 B-like cells

- 14 T-like cells

C 500 SínASE3/RNA SPINK2
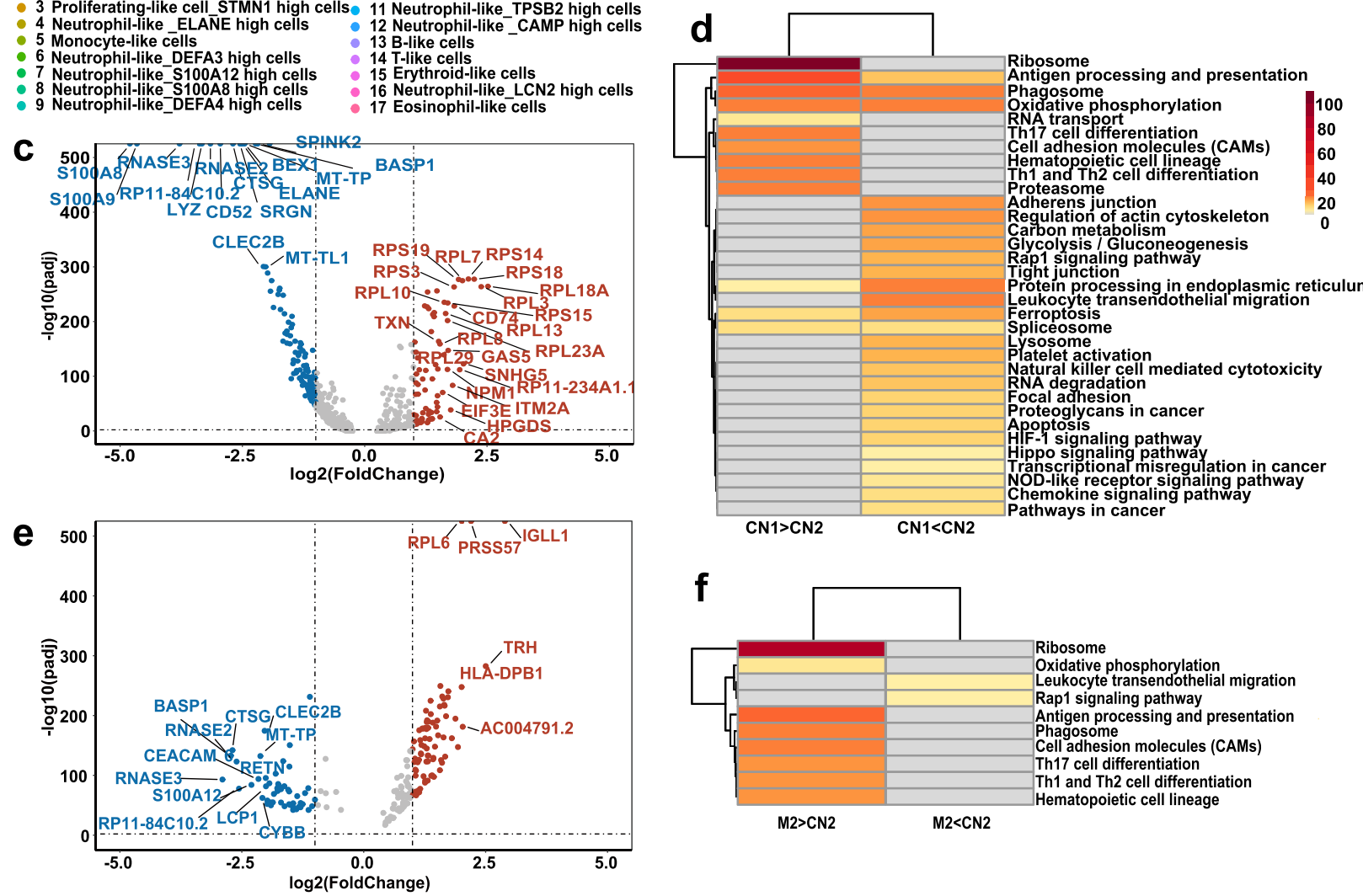

g

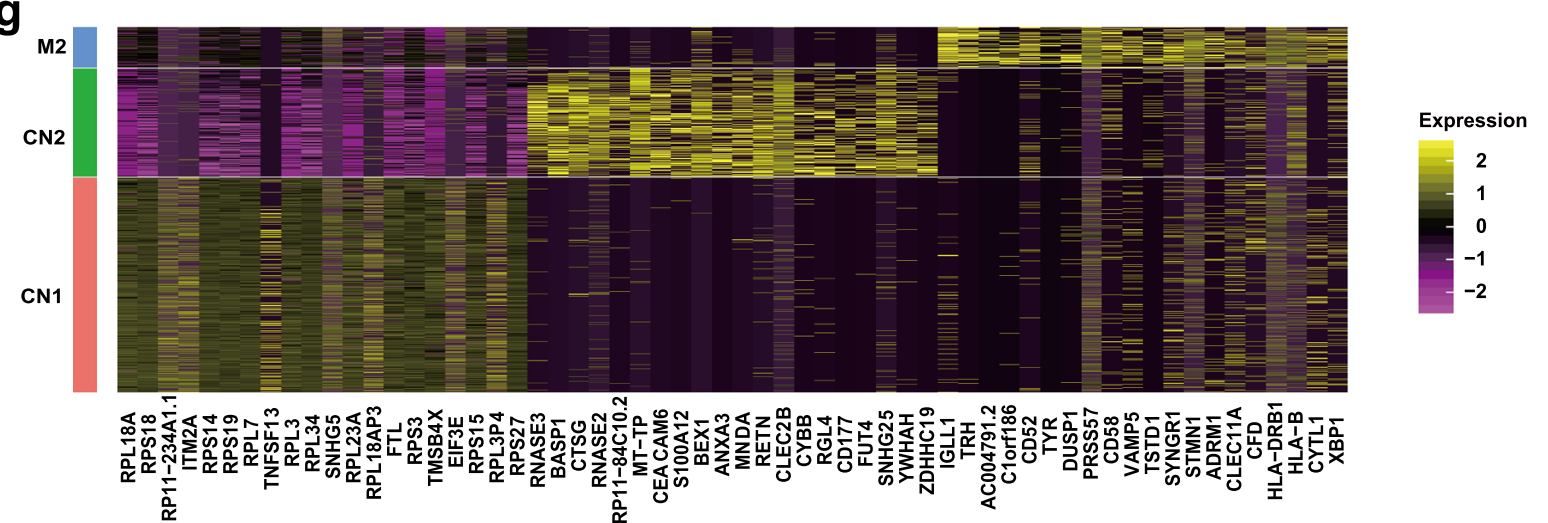

Fig. 2 (See legend on next page.) 
(See figure on previous page.)

Fig. 2 Distributions of cell clusters and single-cell gene expression patterns of blast-like clusters between CN1 and CN2, CN2 and M2. A Overall tdistributed stochastic neighbor-embedding analysis of bone marrow mononuclear cells from the four samples (NC, CN1, CN2, M2). Clusters indicated by different colors and numbers; cell types indicated by different colors of loops. B Overall $t$-distributed stochastic neighbor-embedding analysis of bone marrow mononuclear cells from the four samples. Samples were indicated by different colors and numbers. C Volcano plot of differentially expressed genes (DEGs) in blast-like cells between CN1 and CN2. The dots on the left represent higher expressed genes in CN2 and those on right represent lower in CN2. D Metascape Kyoto Encyclopedia of Genes and Genomes (KEGG) pathway analysis of enriched terms of DEGs in blast-like cell clusters between CN1 and CN2. Color shows $p$ value. E Volcano plot of DEGs in blast-like cells between CN2 and M2. The dots on the left represent higher expressed genes in CN2 and those on right represent lower in CN2. F Metascape KEGG pathway analysis of enriched terms of DEGs in blast-like cell clusters between CN2 and M2. Color shows $p$ value. G Heat map of DEGs among CN1, CN2, and M2 in blast-like cells. Yellow: higher expression level; red: lower expression level. Samples labeled in different colors. CN: Initial of the MDS-MF $2-3$ patient for single-cell sequencing; CN1: The patient CN at her MDS phase; CN2: The patient CN at her leukemic phase; NC: Normal control; M2: The patient with de novo AML-M

Seventeen cell clusters and eight cell types were identified (Fig. 2A,B). We used "blast-like" cells for the differentially expressed genes (DEGs) and gene enrichment analyses to avoid the influences of other cell types. The top 20 DEGs and their reported functions are listed in Supplementary Table 3 . The top 20 increased genes during leukemic transformation included some AMLrelated genes, such as CD52, SRGN, BEX1, BASP1, SPIN $K 2$, NEAT1, and CEACAM6, and some proinflammatory mediators, such as $S 100$ family genes, RNASE3, and $C Y B B$. In contrast, expression levels of many ribosomal protein genes were decreased (Fig. 2C). The S100 family comprises proinflammatory mediators associated with acute and chronic inflammation and neoplasm metastasis [10]. CYBB can produce superoxide, and trigger mitochondria transfer to stimulate BM stromal cells to form AML blast cells [11]. RNASE3 participates in nucleolysis, cell binding, lipid instability, cytotoxicity, and antibacterial activity [12]. However, none of these genes have previously been linked to leukemic transformation of MDS-MF. Gene enrichment analysis revealed that the upregulated pathways during leukemic transformation mainly contributed to inflammation /oxidation /energy metabolism-related signaling and tumorrelated pathways (Fig. 2D). We also explored the DEGs between $\mathrm{CN} 2$ and $\mathrm{M} 2$ samples to detect the differences between secondary AML and de novo AML (Fig. 2E). Genes with higher expression in CN2 were significantly enriched in leukocyte transendothelial migration and the Rap1 signaling pathway (Fig. 2F). Leukocyte transendothelial migration is an inflammation pathway, while Rap1 acts as a molecular switch involved in many biological processes. S100A12, RNASE3, and CYBB were among the genes with higher expression levels in both $\mathrm{CN} 2$ compared with $\mathrm{CN} 1$, and in $\mathrm{CN} 2$ compared with M2 (Fig. 2G).

In conclusion, this study revealed that MDS-MF with even mild MF had a higher risk of leukemic transformation than MDS without MF, suggesting that MDS-MF should have a different risk classification algorithm and may need special treatment. Inflammatory and oxidation activation may be essential processes, while $S 100$ family genes, RNASE3, and $C Y B B$ might be key genes involved in the leukemic transformation of MDS-MF.

\section{Abbreviations}

MDS: Myelodysplastic syndrome; MF: Myelofibrosis; MDS-MF: MDS with myelofibrosis; IPSS: International Prognostic Scoring System; Int: Intermediate; LTT: Leukemic transformation time; BM: Bone marrow; BMMC: BM mononuclear cell; AML: Acute myeloid leukemia; DEG: Differentially expressed gene; $\mathrm{CN}$ : MDS-MF ${ }_{2-3}$ patient for single-cell sequencing; CN1: Patient CN in MDS phase; CN2: Patient CN in leukemic phase; NC: Normal control; M2: Patient with de novo AML-M

\section{Supplementary information}

The online version contains supplementary material available at https://doi. org/10.1186/s40364-021-00304-w.

Additional file 1 Supplementary File 1. Patients and methods.

Additional file $\mathbf{2}$ Supplementary Table 1. Characteristics of patients and treatment strategies.

Additional file 3 Supplementary Table 2. Marker genes of overall $t$ stochastic neighbor embedding map.

Additional file 4 Supplementary Table 3. Reported tumor-related functions of top-20 differentially expressed genes during leukemic transformation in the MDS-MF M $_{-3}$ patient; and of top-20 differentially expressed genes between the leukemic phase of this $M D S-M_{2-3}$ patient and a de novo AML-M 2 patient.

\section{Acknowledgements \\ This work was supported by the Centre for Stem Cell and Regenerative Medicine, Zhejiang University School of Medicine. The authors thank the patients who participated in the study, their supporters, and the investigators. We also thank International Science Editing (http://www. internationalscienceediting.com) for editing a draft of this manuscript.}

\section{Authors' contributions}

Jie Sun and Guoji Guo designed the study; Minghua Hong interpreted the data, performed the statistical analysis and wrote the manuscript; Junqing Wu, Lifeng Ma and Xiaoping Han performed single-cell sequencing and bioinformatics analyses; Zhaoming Wang and Jing Zhao re-evaluated and graded the myelofibrosis in all the patients; Huanping Wang performed the FISH tests; Zhimei Chen performed karyotype analysis; Ting Lu, Lizhen Liu, Huarui Fu, Weijia Huang, Weiyan Zheng, Jingsong He, Guoqing Wei collected the clinical data and provided valuable biological specimens, He Huang and Zhen Cai supervised the clinical work. The author(s) read and approved the final manuscript.

\section{Funding}

This study was funded by the National Nature Science foundation of China (82070200) and The Nature Science Foundation of Zhejiang Province 
(LQ20H160025). The funding bodies did not participate in the study design data collection, analysis, interpretation, or writing of the manuscript.

\section{Availability of data and materials}

The data and materials will be available upon corresponding author approval. All data sets generated/analyzed for this study are included in the manuscript and the additional files.

\section{Declarations}

\section{Ethics approval and consent to participate}

Informed consent was obtained from all patients and the protocol was approved by the Ethics Committee of the First Affiliated Hospital of Zhejiang University.

\section{Consent for publication}

All authors agreed to publish.

\section{Competing interest}

The authors declare that the research was conducted in the absence of any commercial or financial relationships that could be construed as potential competing interests.

\section{Author details}

${ }^{1}$ Bone Marrow Transplantation Center, the First Affiliated Hospital, Zhejiang University School of Medicine; Institute of Hematology, Zhejiang University; Zhejiang Province Engineering Laboratory for Stem Cell and Immunity Therapy; Liangzhu Laboratory, Zhejiang University Medical Center, 1369 West Wenyi Road, 310003 Hangzhou, China. ${ }^{2}$ Center for Stem Cell and Regenerative Medicine, Stem Cell Institute, School of Medicine, Zhejiang University, 310058 Hangzhou, China. ${ }^{3}$ Pathology Department, the First Affiliated Hospital, School of Medicine, Zhejiang University, 310003 Hangzhou, China.

Received: 19 March 2021 Accepted: 26 May 2021

Published online: 02 July 2021

\section{References}

1. Marisavljević D, Rolović Z, Cemerikić V, Bosković D, Colović M. Myelofibrosis in primary myelodysplastic syndromes: clinical and biological significance. Medical oncology (Northwood, London, England). 2004;21:325-331.

2. Buesche G, Teoman H, Wilczak W, Ganser A, Hecker H, Wilkens L, et al. Marrow fibrosis predicts early fatal marrow failure in patients with myelodysplastic syndromes. Leukemia. 2008;22:313-22.

3. Kroger N, Zabelina T, van Biezen A, Brand R, Niederwieser D, Martino R, et al. Allogeneic stem cell transplantation for myelodysplastic syndromes with bone marrow fibrosis. Haematologica. 2011;96:291-7.

4. Tefferi A, Vaidya R, Caramazza D, Finke C, Lasho T, Pardanani A. Circulating interleukin (IL)-8, IL-2R, IL-12, and IL-15 levels are independently prognostic in primary myelofibrosis: a comprehensive cytokine profiling study. Journal of clinical oncology: official journal of the American Society of Clinical Oncology. 2011;29:1356-63.

5. Mesa R, Hanson C, Rajkumar S, Schroeder G, Tefferi A. Evaluation and clinical correlations of bone marrow angiogenesis in myelofibrosis with myeloid metaplasia. Blood. 2000;96:3374-80.

6. Koschmieder S, Mughal T, Hasselbalch H, Barosi G, Valent P, Kiladjian J, et al. Myeloproliferative neoplasms and inflammation: whether to target the malignant clone or the inflammatory process or both. Leukemia. 2016;30: 1018-24.

7. Bjørn M, Hasselbalch $H$. The Role of Reactive Oxygen Species in Myelofibrosis and Related Neoplasms. Mediat Inflamm. 2015;2015:648090.

8. Wu J, Xiao Y, Sun J, Sun H, Chen H, Zhu Y, et al. A single-cell survey of cellular hierarchy in acute myeloid leukemia. J Hematol Oncol. 2020;13:128.

9. Han X, Zhou Z, Fei L, Sun H, Wang R, Chen Y, et al. Construction of a human cell landscape at single-cell level. Nature. 2020;581:303-9.

10. Wang S, Song R, Wang Z, Jing Z, Wang S, Ma J. S100A8/A9 in Inflammation. Frontiers in immunology. 2018:9:1298

11. Marlein CR, Zaitseva L, Piddock RE, Robinson SD, Edwards DR, Shafat MS, et al. NADPH oxidase-2 derived superoxide drives mitochondrial transfer from bone marrow stromal cells to leukemic blasts NADPH. Blood. 2017;130: $1649-60$.
12. Lien PC, Kuo PH, Chen CJ, Chang HH, Fang SL, Wu WS, et al. In silico prediction and in vitro characterization of multifunctional human RNase3. Biomed Res Int. 2013;2013:170398.

\section{Publisher's Note}

Springer Nature remains neutral with regard to jurisdictional claims in published maps and institutional affiliations.
Ready to submit your research? Choose BMC and benefit from:

- fast, convenient online submission

- thorough peer review by experienced researchers in your field

- rapid publication on acceptance

- support for research data, including large and complex data types

- gold Open Access which fosters wider collaboration and increased citations

- maximum visibility for your research: over $100 \mathrm{M}$ website views per year

At $\mathrm{BMC}$, research is always in progress.

Learn more biomedcentral.com/submissions 